\section{Cahiers de Narratologie}

Analyse et théorie narratives

39 | 2021

L'héritage de Ricoeur : du récit à l'expérience

\title{
Françoise Lavocat. Les Personnages rêvent aussi.
}

\section{Marie-Laure Ryan}

\section{(2) OpenEdition}

Journals

Édition électronique

URL : https://journals.openedition.org/narratologie/12150

DOI : 10.4000/narratologie. 12150

ISSN : 1765-307X

Éditeur

LIRCES

Référence électronique

Marie-Laure Ryan, «Françoise Lavocat. Les Personnages rêvent aussi. », Cahiers de Narratologie [En ligne], 39 | 2021, mis en ligne le 11 juin 2021, consulté le 21 juillet 2021. URL : http://

journals.openedition.org/narratologie/12150; DOI : https://doi.org/10.4000/narratologie.12150

Ce document a été généré automatiquement le 21 juillet 2021.

Article L.111-1 du Code de la propriété intellectuelle. 


\title{
Françoise Lavocat. Les Personnages rêvent aussi.
}

\author{
Marie-Laure Ryan
}

\section{RÉFÉRENCE}

Hermann éditeurs, collection Fiction Pensantes. 2020.

1 La théorie et la fiction demandent des talents différents; il est rare qu'un auteur excelle dans les deux genres (exceptions notoires : Umberto Eco et David Lodge). Il est encore plus rare qu'un même texte réussisse à stimuler à la fois l'imagination, par sa dimension fictionnelle, et la réflexion théorique. C'est cette gageure que tient Françoise Lavocat avec Les Personnages rêvent aussi.

2 L'ouvrage entraine le lecteur dans une expédition sur la planète Fiction, appartenant à un autre système solaire que la Terre, et plus précisément dans une ville nommée Shadavar qui tient son nom d'un gracieux quadrupède à la corne unique et où sont réunis « tous les personnages inventés sur Terre en provenance des romans, des contes de fées, du cinéma, des chansons, du théâtre, des tableaux, des religions auxquelles on ne croit plus » (9). Le livre mentionne plus de 400 personnages, dont un répertoire est donné à la fin du livre, avec des résumés de leur fiction d'origine et des notes sur leurs adaptations dans d'autres médias. A côté des inévitables célébrités littéraires, comme Don Quichotte, Schéhérazade et Madame Bovary, cette liste comprend des stars de la culture populaire, comme le Parrain Vito Corleone, Hermione Granger d'Harry Potter et Lynette Scavo de Desperate Housewives, ainsi que des prima donnas d'opéra comme Dorabella et Fiordiligi de Cosi Fan Tutte. Les auteurs (Flaubert, Pirandello, Molière) ne sont représentés que s'ils apparaissent dans une fiction. Il y a d'autres villes sur la planète Fiction pour les personnages de cultures non occidentales, et d'autres planètes dans la galaxie des mondes possibles pour les personnages des religions auxquelles on croit encore ou pour les personnages historiques, mais un constant va-et-vient permet 
aux habitants de ces autres villes et planètes d'immigrer à Shadavar, comme c'est le cas pour les héros de la mythologie gréco-romaine.

3 En dépit de ce mouvement d'immigration, Shadavar n'accepte pas n'importe qui car les conditions d'admission sont devenues de plus en plus strictes avec le temps. Au Moyenâge ou à la Renaissance tout le monde était admis parce que le concept de fiction n'était pas encore l'objet d'une réflexion théorique: dans le Roland Furieux de l'Arioste, par exemple, des personnages bibliques, mythologiques, et historiques cohabitent avec des créatures fantastiques. Mais de nos jours les personnages doivent passer par une machine nommée Chiméramètre qui mesure leur pureté ontologique pour devenir citoyens de la planète Fiction ; cela soulève de sérieux problèmes pour les héros d'autofictions et pour ceux de romans historiques. Cette machine, on le suppose, peut être reprogrammée pour refléter l'importance de la notion de fictionnalité à diverses époques et dans diverses cultures. Une condition d'admission typique de l'esthétique postmoderniste est qu'un personnage doit manifester un certain degré d'autoréflexivité (18). Cela peut se comprendre comme la capacité d'un personnage d'inspirer une réflexion sur la nature de la fictionnalité ; mais si on interprète cette condition littéralement comme l'admission par un personnage de sa propre fictionnalité, Shadavar ne serait peuplé que de "métaleptiques » ou de personnages désespérément en quête d'auteur, excluant les héros des romans réalistes, comme Emma Bovary et Mr Pickwick, qui sont les vedettes de Shadavar.

4 L'intrigue de l'ouvrage se noue entre deux personnages menacés de disparition, car si la planète Fiction accepte en principe tous les personnages jamais crées, elle ne les garde qu'aussi longtemps qu'ils vivent dans l'imagination humaine; quand plus personne ne s'intéresse à eux, ils deviennent des fantômes diaphanes, ni morts ni vivants, et incapables d'action. L'un de ces deux personnages en danger est une métaleptique, Cecilia, l'héroïne du film de Woody Allen La Rose pourpre du Caire, qui tombe amoureuse du héros de La Rose pourpre du Caire, un film-dans-le-film, et entretient une liaison aussi bien avec lui qu'avec l'acteur qui l'incarne. Pourquoi est-elle menacée de disparition? Parce qu'en un âge de pudibonderie, la conduite pas toujours édifiante de son auteur suscite aux Etats-Unis l'exclusion de ses films des curriculums universitaires. Cette tendance actuelle contraste avec la soi-disant «mort de l'auteur» de l'époque structuraliste, ou avec la prédilection dans les années cinquante et soixante pour les auteurs en rupture de justice comme Sade et Genet. L'autre héros de l'ouvrage est le prince Fan-Feredin, inventé à la fin du XVIIIème siècle par un jésuite nommé Guillaume-Hyacinthe Bougeant, « qui n'était pas un romancier et qui voulait montrer, à travers les aventures de Fan-Feredin, la sottise et la nocivité des romans » (31). Avec un tel programme il n'est pas étonnant que Fan-Feredin ait sombré dans l'oubli, mais son roman a été réédité en 1992, et depuis qu'il est disponible en ligne, il jouit d'un certain succès dans un public universitaire. Il y a aussi un personnage maléfique, Herman Sororis, dont la fiction d'origine n'est autre que Les Personnages rêvent aussi et dont la spécialité est de mettre en scène des opéras qui transportent les personnages dans des milieux entièrement différents de leur lieu et leur temps d'origine.

L'existence de multiples versions d'un même personnage soulève un problème philosophique que l'auteur aborde en s'appuyant sur la théorie des mondes possibles. Deux interprétations sont proposées : la doctrine Kripkéenne (d'après Saul Kripke et sa théorie de la désignation rigide), qui maintient qu'à chaque nom ne correspond qu'un seul individu, en dépit des variations de cet individu dans divers mondes possibles, de 
sorte que tous les Don Quichotte, Sherlock Holmes et autres Don Juan ne font qu'un à Shadavar ; et la doctrine Russellienne, basée sur la notion de description définie, qui conçoit les référents des noms comme des ensemble de propriétés, de sorte que toutes les versions de Don Quichotte ou Don Juan correspondent à des entités différentes. La doctrine russellienne mène à la surpopulation de Shadavar, alors que la doctrine kripkéenne conduit à une excessive réduction, et rencontre des difficultés à distinguer l'empereur Napoléon d'un chien nommé Napoléon. Il y a aussi la doctrine de David Lewis, qui affirme qu'il n'y a qu'une façon d'exister et que tous les personnages sont réels, et se considèrent comme tels, dans le monde de leur fiction, sauf évidemment ceux qui se disent fictionnels - paradoxe insolvable pour la logique. Une telle doctrine interdit la cohabitation des personnages issus de divers textes sur la planète Fiction puisqu'elle lie leur identité à leur monde d'origine ; mais ils ont des alter egos dans d'autres mondes qui eux aussi se considèrent comme réels. L'Emma Bovary de Shadavar ne serait donc pas celle de Flaubert, mais celle de Françoise Lavocat bien qu'elles partagent (plus ou moins) une même destinée. Pour résumer ces chicaneries philosophiques, il n'y a pas de solution idéale au problème des incarnations multiples ; mais il y a une solution technologique. Celle-ci consiste à réduire les versions multiples d'un personnage en une seule incarnation en les faisant passer par un «synthétiseur de versions ».

6 De nombreux problèmes théoriques sont abordés au cours de dialogues entre Cecilia et Fan-Feredin, auxquels participent aussi Jill, l'hérö̈ne d'un autre film de Woody Allen, et Emma Bovary qui révèle une acuité d'esprit bien supérieure à ce qu'elle démontre dans le roman de Flaubert. L'un de ces problèmes - préoccupation majeure pour la narratologie cognitive-- concerne les raisons de notre amour pour la fiction. Jill, une étudiante passablement bas-bleu, expose avec diligence la théorie. Fan-Feredin, créé par un jésuite, en appelle à la dimension pédagogique de la fiction. Elle nous enseigne des valeurs morales, nous apprend à partager les émotions des personnages, nous invite à formuler des jugements éthiques sur leur conduite et nous révèle l'expérience d'êtres que nous n'aurions pas l'occasion de rencontrer dans notre vie. Mais Cecilia n'a cure de ces explications. Sa passion pour un personnage de film va à l'encontre de tout ce que nous enseigne la théorie. Comme l'écrivait Pascal, l'amour a ses raisons que la raison ne connait pas.

7 Mais revenons à l'intrigue. Elle est à vrai dire difficile à suivre, vu le nombre et la diversité des personnages, et son intérêt réside dans leurs palabres et non dans leurs actions. Fan-Feredin est amoureux de Cecilia et la cherche sans succès dans une géographie instable ou toutes les régions communiquent entre elles, ce qui rend impossible la classification des personnages selon des critères comme les genres, les médias, ou les périodes historiques. Il s'avère que Cecilia a été kidnappée, ainsi que ses amants Tom et Gil, par un sinistre trio composé de Raoul Hirsch, le metteur en scène de La Rose pourpre du Caire (le film dans le film), l'Architecte de Matrix qui conçoit le remplacement du réel par une simulation, et Vautrin de Balzac. Ces personnages se sont servis du "réflecteur d'ondes", une technologie qui assure la communication entre la terre et la planète Fiction, pour faire entrer Cecilia et ses amants dans un jeu vidéo ou ils interagissent littéralement avec des humains. C'est d'ailleurs Vautrin qui a eu l'idée d'assurer la survie de quelques 2350 personnages de la Comédie Humaine menacés d'extinction (car on ne lit plus guère qu'une demi-douzaine des romans de Balzac), en faisant d'eux des avatars de jeux vidéo. Mais s'il existe des usages légitimes du réflecteur d'onde - ainsi que le démontrent les milliers de femmes qui adoptent le 
costume de l'héroïne du Handmaid Tale de Margaret Atwood pour protester contre la suppression de leurs droits de reproduction - cette technologie ne doit en aucun cas être utilisée pour faire passer les personnages dans le réel, ni pour que des personnes réelles se prennent sérieusement pour les personnages ou les avatars qu'ils créent, comme semble le faire l'auteure française Chloé Delaume (167). Un procès s'ensuit, à l'issue duquel Vautrin, Raoul Hirsch et l'Architecte sont condamnés, et le trio Cecilia, Tom et Gil autorisé à retourner dans la planète Fiction, après avoir subi une cure d'amélioration des avatars. Tous les avatars de jeux passent aussi par cette cure, mais pour la plupart d'entre eux le traitement est un échec total, car ils n'ont pas la substance nécessaire pour survivre sur la planète Fiction: «Les [vrais] personnages font rêver, penser, permettre de s'identifier, que sais-je. Les avatars servent essentiellement à jouer, même s'ils ressemblent à des personnages » (178). On pourrait évidemment citer certains personnages de jeux, comme Lara Croft de Tomb Raider, Sonic the Hedgehog, ou les Super Mario Brothers, qui ont inspiré des films; mais ces films sont en général médiocres, et seront beaucoup plus vite oubliés que les jeux qui les inspirent. Par contre la cure réussit pour le trio de Cecilia, Tom et Gil : après un séjour sur la montagne magique de Thomas Mann, ils sont réintégrés à Shadavar.

Si Les Personnages rêvent aussi ignore les frontières entre genres, périodes et mondes fictionnels, ce «salmigondis ontologique» (167) présente une conception de la fictionnalité qui maintient la distinction entre personnes et personnages. Les métaleptiques comme Cecilia peuvent passer d'un monde à l'autre à l'intérieur de leur fiction, mais ils ne peuvent pas transgresser la frontière entre la fiction et le réel. $\mathrm{A}$ l'intérieur de leur fiction, les personnages les plus réussis sont toutefois si vivants qu'ils permettent au lecteur ou spectateur de parler d'eux comme s'ils étaient citoyens de la planète Terre. De Marietta, un autre personnage de Woody Allen, l'auteure (ou narrateur ?) écrit : "Elle est charmante mais elle se mêle de ce qui ne la regarde pas " (86). De tels ragots horrifieraient les textualistes qui conçoivent les personnages comme des collections de sèmes, mais ils saisissent le ton frondeur d'une «fiction pensante » non seulement impressionnante d'érudition mais aussi pétillante d'esprit et d'invention.

\section{AUTEUR}

\section{MARIE-LAURE RYAN}

Chercheure indépendante, Colorado, USA 